\title{
La práctica docente como actividad creativa de equilibrio entre la planeación y la intuición
}

\author{
Alejandra Torres Landa López
}

Fecha de recepción: 08 de julio de 2019 Fecha de aceptación: 10 de septiembre de 2019

\section{Resumen}

E ste texto aborda el tema de la creatividad en la práctica docente, comenzando con la diferenciación entre creatividad y diseño y, a su vez, cómo éstos deben ser complementarios. Se plantean algunos enfoques en la planeación e implementación de asignaturas, así como problemas en los que se vislumbran posibles barreras para la creatividad en la práctica docente. Más adelante, se proponen algunas soluciones, con el fin de fomentar la creatividad en el salón de clases, desde la planeación de la asignatura, el uso de la intuición e improvisación en la labor docente como fase creativa del profesor, para cerrar con instrumentos creativos de evaluación permitiendo, de esta manera, concluir con algunas reflexiones finales.

Palabras clave: creatividad en la docencia, profesor creativo, enseñanza creativa.

\section{Introducción}

\section{Creatividad no es sinónimo de diseño, ${ }^{1}$ pero son complementarios}

La creatividad es una actividad constante e inherente al ser humano, a diferencia del diseño, que es un ejercicio sistemático con un propósito específico que además permite una metacognición del proceso creativo, es decir, que permite tener consciencia de la creación. Por una parte, se considera a la creatividad como la habilidad de transformar ideas tradicionales; relacionada con la originalidad, progreso e imaginación: "El término creatividad significa innovación valiosa" (Mesanza, 1999: 333).

Algunas de las características del pensamiento creativo son: 1) diferentes a los demás; 2) creadores de una amplitud de categorizaciones, es decir, que las personas creativas tiendan a sacar conclusiones más amplias; 3 ) pensamientos fluidos, lo que produce una enorme cantidad

1 Considerado como la actividad creativa del ser humano que tiene por misiva proyectar objetos o procesos útiles, como en este caso la planeación del proceso de enseñanza y aprendizaje. de ideas; 4) hábiles para transferir ideas rápidamente de una categoría a otra, mostrando una flexibilidad de pensamiento; y 5) originales, donde las ideas son decisiones independientes, inusuales e ingeniosas (Fidelman, 2008). Por otra parte, se puede señalar que el diseño es un método para resolver problemas y crear nuevos conocimientos (Polo \& Polo, 2015); además, se trata de un proceso creativo que responde a una necesidad en particular (Wong, 2001; Scott, 1995).

El sistema educativo, ya sea nivel básico, medio superior o superior, diseña actividades educativas con el propósito de lograr objetivos específicos de aprendizaje. La responsabilidad de las instituciones educativas (IE) sería, además de lograr los objetivos disciplinares, ayudar a los estudiantes a desarrollar su propia creatividad en cada una de sus etapas de formación; sin embargo, una de las formas de lograrlo consiste en que el propio proceso educativo sea creativo. Desafortunadamente, en muchas ocasiones, las escuelas no sólo entorpecen la creatividad, sino que la inhiben (Robinson, 2006). 


\section{El problema}

\section{Enfoques para la planeación \\ e implementación de asignaturas}

En las instituciones de educación superior (IES), el primer problema que se identifica en la planeación del proceso educativo es el enfoque pedagógico utilizado, ya que "pedagogía es el arte de educar a los niños" (Mesanza, 1999: 1078), así que, si en las universidades se sigue tratando a los estudiantes como niños, continuarán comportándose como tal. Por ello, las IEs deberían enseñar bajo un enfoque andragógico, pues refiere a la ciencia de la educación del adulto (Mesanza, 1999), de tal manera que se puedan aprovechar las características del estudiante adulto, como: 1) la autonomía; 2) las experiencias previas; 3 ) el interés y motivación por el área de estudio; 4) que la mayoría se encuentren insertos en la vida laboral -lo cual beneficiará para que el aprendizaje sea significativo-; y 5) puedan organizar mejor sus tiempos (Torres \& Martínez, 2017).

Ahora bien, utilizar la andragogía en el proceso educativo responde al Modelo Educativo Institucional (MEI) de la Universidad Autónoma de Aguascalientes (UAA), el cual, al igual que en la mayoría de las IES, promueve un modelo constructivista, donde el estudiante es el centro del proceso educativo y constructor de su propio conocimiento, mientras el profesor lo ayuda a aprender, reconociendo los conocimientos previos, siendo, al mismo tiempo, acompañamiento y guía durante todo su proceso de aprendizaje (UAA, 2007).

Otro de los problemas identificados es la repetición de viejos patrones, es urgente cambiar de paradigma educativo, debido a que los modelos actuales responden a necesidades del pasado, específicamente a capacitar a obreros para la época de la Revolución Industrial (Robinson, 2010), debido a que la sociedad posmoderna del siglo XXI requiere enfoques diferentes (Lyotard, 1999).

\section{Una solución}

\section{La creatividad en la práctica docente}

Lo anterior permite catalogar como indispensable la existencia de una transformación constante en el quehacer diario del maestro, ya que la educación es una secuencia continua de actividades destinadas al aprendizaje de los estudiantes. No obstante, cada estudiante es diferente; además, cambia el ambiente, el momento social y otros elementos adyacentes, los cuales definen características especiales que vuelven imperativo el cambio en las dinámicas para ajustarse a las necesidades en cada sesión.

A pesar de que la creatividad debe estar presente en cada momento de la práctica docente, se identifican tres momentos específicos: 1) la planeación, 2) la implementación y 3) la evaluación.

\section{《 Una de las formas para lograr que los} estudiantes desarrollen su propia creatividad es que el propio proceso educativo sea creativo. $\gg$

\section{La creatividad en la planeación de una asignatura}

La UAA ofrece un formato para la planeación y seguimiento que ayuda al profesor a planear todo un periodo, lo que se ha convertido en una práctica común entre los profesores. Este instrumento le facilita al profesor diseñar los planes de clase, es decir, el día a día de su quehacer docente. La creatividad en la planeación consiste en proponer diferentes dinámicas para que el estudiante aprenda las temáticas de la asignatura, con el fin de atender los diferentes estilos cognitivos ${ }^{2}$ (Arregui, 1999), mismos que derivan en estilos de aprendizaje, ${ }^{3}$ por lo que la planeación debería variar en estrategias de enseñanza ${ }^{4}$ y ambientes de aprendizaje (Aduna, 2004).

Por otra parte, los profesores deben tomar en consideración que los estudiantes son nativos de la tecnología, es por ello que utilizar las tecnologías de información y comunicación (TIC) en distintas actividades, promueve el aprendizaje significativo de los estudiantes. Además de incorporar las TIC en clases tradicionales, es recomendable utilizarlas para crear cocteles educativos (Torres Landa, 2014); es decir, aunado a utilizar tecnología en clases presenciales, se deben combinar los ambientes frente al grupo con actividades en línea, de tal manera que se utilice lo mejor de los dos ámbitos (tanto presenciales como en

2 Dependencia/independencia; reflexividad/impulsividad; simple/ complejo; flexible/rígido, etcétera (Arregui, 1999).

3 Mismos que se pueden clasificar según: 1) el modelo de los cuadrantes cerebrales de Herrmann: cortical izquierdo, cortical derecho, límbico izquierdo, límbico derecho; 2) el modelo de Felder y Silverman: sensitivo-intuitivo, visual-verbal, inductivodeductivo, secuencial-global, activo-reflexivo; 3) el modelo de Kolb: teórico, pragmático, reflexivo, activo; 4) el modelo de inteligencias múltiples de Gardner: intrapersonal, interpersonal, musical, espacial, corporal-kinética, lógico-matemática, lingüística; 5) el modelo de programación neurolingüística de Bandler y Grinder: auditivo, visual, kinestésico; 6) el modelo de hemisferios cerebrales: derecho, izquierdo (Aduna, 2004).

4 Tales como: aprendizaje multisensorial, fantasía, experiencia directa, pensamiento visual, metáfora, entre otras (Aduna, 2004). 
línea), logrando ambientes combinados que enriquezcan el proceso de enseñanza y aprendizaje e, incluso, poder emigrar a los ambientes $100 \%$ en línea. La planeación previa ayudará a identificar el mejor ambiente y estrategia que responda a las necesidades temáticas de la asignatura.

\section{La intuición e improvisación en clase es parte de la fase creativa del profesor}

A pesar de que la planeación de la clase es indispensable y una actividad diaria del profesor, es evidente la existencia de eventos que ocurren durante una sesión, los cuales obligan al cambio de estrategia en ese preciso momento, para lo cual el profesor debe valerse de su intuición, proceso en donde la persona codifica, clasifica $\mathrm{y}$ accede de manera eficiente a modelos mentales, concebidos por sus conocimientos y experiencias previas, con el fin de utilizarlos en la toma de decisiones para el cambio. Dicho de otra manera, el profesor tiene esquemas cognitivos que nacen de la experiencia y le permiten superponerse a problemas particulares y proponer soluciones oportunas (Weimer, 2013).

Se habla de intuición, pero la improvisación también es un elemento clave que permite al profesor ser creativo en clase; improvisación concebida como la acción llevada a cabo en un momento determinado sin "planeación previa", sin un "guion" donde se prevea paso a paso cada movimiento. Se refiere a la improvisación del latín improvisus, cuyo significado es "imprevisto"; no a la idea de $\ll$ Es indispensable que en el quehacer diario del maestro exista una transformación constante. La creatividad debe estar presente

en cada momento de la práctica docente: planeación, implementación y evaluación. $\gg$

improvisación de hace algún tiempo, en la cual el profesor podía llegar a clase y decirles a los estudiantes que todos aprenderían juntos, o no sabía ni siquiera el tema que daría en esa sesión. Como lo menciona el MEI de la UAA, el profesor debe ser un profesional con pleno dominio de su área de conocimiento, un educador con la capacidad de planificar su quehacer docente de manera sistemática, generando distintos ambientes de aprendizaje, adaptando la enseñanza a las necesidades y ritmo de los estudiantes, siendo un promotor de valores, entre otros (UAA, 2007).

\section{La evaluación también debe ser creativa}

Para concluir el ciclo del proceso educativo, es indispensable considerar igualmente la evaluación, al ser una dimensión de dicho proceso (UAA, 2007). Debe ser también diseñada de manera creativa, así como tomar de referencia los elementos esenciales de la evaluación (Morales, Ramos \& Torres, 2018), donde es necesario variar el tipo de instrumento utilizado: exámenes, trabajos, ensayos, mapas conceptuales, el juego, entre otros, y la forma de presentarlo.

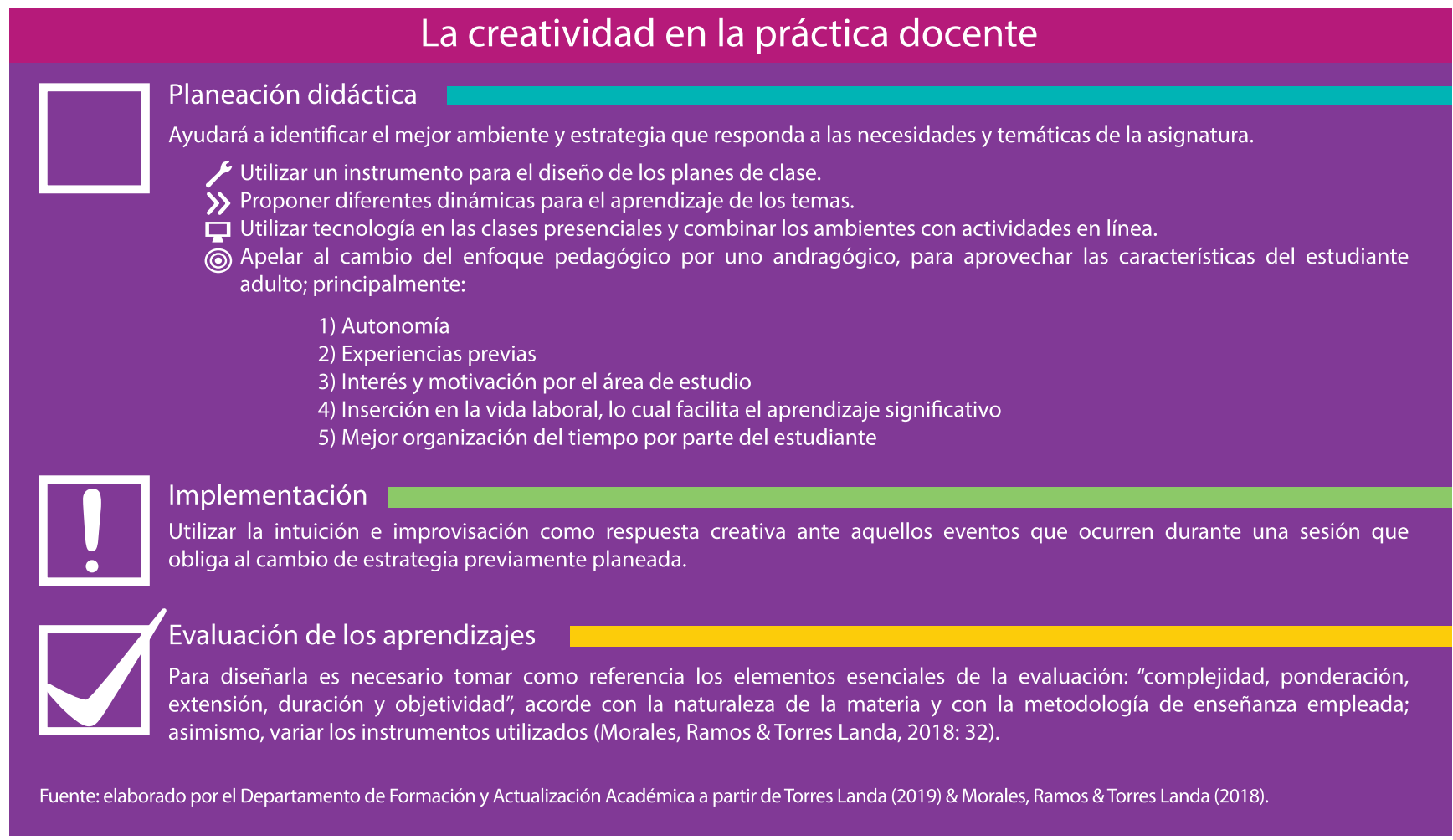




\section{Conclusión}

La creatividad en la práctica docente debe estar presente en todos los momentos del proceso educativo, desde la planeación del cronograma de una asignatura, al diseñar las estrategias de enseñanza y proponer las actividades de aprendizaje para los estudiantes; hasta el diseño del plan de clase o sesión, incluyendo la intuición e improvisación para responder a situaciones que se dan en el momento y en la evaluación, tanto de los aprendizajes de los estudiantes como del propio proceso, de tal manera que surjan evidencias que permitan mejorar la experiencia educativa.

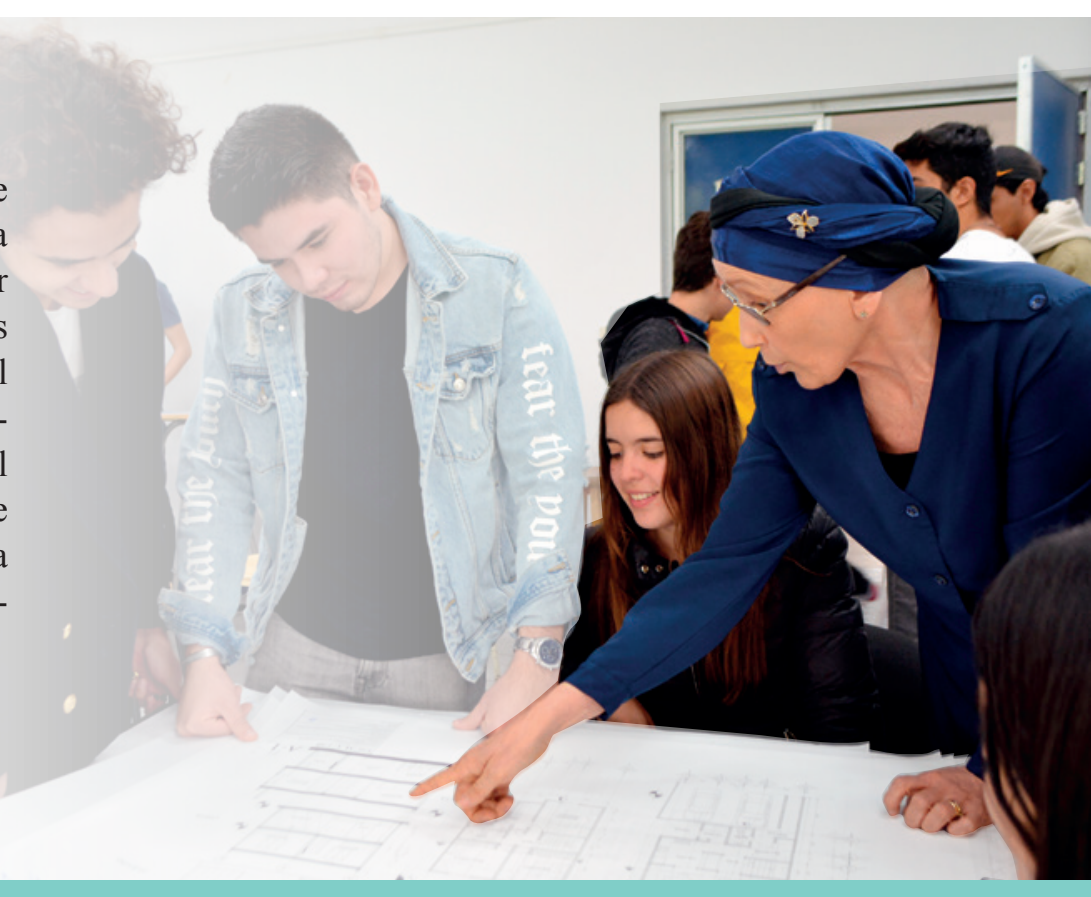

\section{Fuentes de consulta}

Aduna, A. (Coord.). (2004). Manual de estilos de aprendizaje. México: SEP. Disponible en: https://bit. ly/1m1lHKD [Consultado en junio de 2019].

Arregui, M. (1999). Enciclopedia general de la educación. España: Océano.

Fidelman, M. (2008). Two different paths to creativity. Electronic Journal of Research in Educational Psychology, 6(1), 1-27. Disponible en: https://bit.ly/35IfS02 [Consultado en junio de 2019].

Lyotard, J. (1999). La condición postmoderna: informe sobre el saber. España: Cátedra.

Mesanza, J. (Coord.). (1999). Diccionario de las ciencias de la educación. México: Santillana.

Morales, A., Ramos, G. \& Torres Landa, A. (2018). Protocolo para el diseño de exámenes extraordinarios. DOCERE, año 9, (19), 28-30.

Polo, R. \& Polo, D. (2015). ¿Necesitamos los diseñadores una teoría del diseño? ¿Nos hace falta una retórica unificadora? Iconofacto, 11(17), 54-72.

Robinson, K. (2006). Las escuelas matan la creatividad [Video]. YouTube. Disponible en: https://bit. ly/2Ne13vL [Consultado en junio de 2019].

Robinson, K. (2010). RSA ANIMATE: Changing education paradigms [Video]. YouTube. Disponible en: https:// www.youtube.com/watch? $=z D Z F c D G p L 4 U$ [Consultado en junio de 2019].

Scott, R. (1995). Fundamentos del diseño. México: Limusa.
Torres Landa, A. (2014). Blended learning, an "educational cocktail"; social interaction in a mixture of learning environments [Video]. YouTube. Disponible en: https://www.youtube.com/watch?v=BIOuLvGgSPg [Consultado en junio de 2019].

Torres Landa, A. (2019). La práctica docente como actividad creativa de equilibrio entre la planeación y la intuición. DOCERE, año 10, (21), 11-14.

Torres Landa, A. \& Martínez, E. (2017). Lo mejor de dos mundos: los ambientes combinados favorecen el modelo constructivista para aprendizajes significativos de la arquitectura. En S. Kassis y S. Carrión (Comp.). Procesos de enseñanza-aprendizaje en las escuelas de arquitectura ante el avance tecnológico del siglo XXI (pp. 257-262). México: Asinea. Disponible en: https://bit.ly/2OSIKOt [Consultado en junio de 2019].

UAA. (2007). Modelo Educativo Institucional. Correo Universitario, séptima época, (15) [1 ${ }^{\mathrm{a}}$. reimp.], 29 de mayo de 2015. México: Universidad Autónoma de Aguascalientes.

Weimer, M. (29/mayo/2013). Understanding the Role of Intuition in Teaching. Faculty Focus. Disponible en: https://www.facultyfocus.com/articles/teachingcareers/understanding-the-role-of-intuition-in-teaching/ [Consultado en junio de 2019].

Wong, W. (2001). Fundamentos del diseño (3a. ed.). España: G. Gili. 